Archives

18-19 | 1997

Inventaire des archives de Robert Mandrou

\title{
Inventaire sommaire
}

Brigitte Mazon et Marie-Annick Morisson

\section{(2) OpenEdition \\ Journals}

\section{Édition électronique}

URL : http://journals.openedition.org/ccrh/2550

DOI : $10.4000 /$ ccrh. 2550

ISSN : $1760-7906$

Éditeur

Centre de recherches historiques - EHESS

Édition imprimée

Date de publication : 10 octobre 1997

ISSN : 0990-9141

Référence électronique

Brigitte Mazon et Marie-Annick Morisson, «Inventaire sommaire », Les Cahiers du Centre de Recherches Historiques [En ligne], 18-19 | 1997, mis en ligne le 20 février 2009, consulté le 30 avril 2019. URL : http://journals.openedition.org/ccrh/2550; DOI : 10.4000/ccrh.2550

Ce document a été généré automatiquement le 30 avril 2019

Article L.111-1 du Code de la propriété intellectuelle. 


\title{
Inventaire sommaire
}

\author{
Brigitte Mazon et Marie-Annick Morisson
}

\section{Papiers personnels (1932-1986)}

Ces dossiers rassemblent les pièces officielles et administratives personnelles de Robert Mandrou. Il est à noter qu'un ensemble de documents familiaux, qui sera versé avec le fonds aux Archives nationales, ne figure pas dans l'inventaire ici présenté.

\section{Éléments biographiques}

Curriculums vitae établis à différents moments de la carrière.

\section{Pièces d'état civil (1942-1982)}

Cartes d'identité, passeports, différentes cartes d'associations.

Documents militaires (essentiellement le STO, 1942-1943), documents concernant l'association des déportés du travail (attribution du titre de "personne contrainte au travail en pays ennemi »), 1943-1962.

\section{Carnets d'adresses et agendas (1954-1976)}

\section{Diplômes (originaux) (1940-1983)}

Diplôme de bachelier de l'enseignement secondaire, 20 mai 1940.

Diplôme d'Officier dans l'Ordre des Palmes académiques, 24 août 1976.

Diplôme de Commandeur dans l'Ordre des Palmes académiques, 9 octobre 1981.

Diplôme de Chevalier de l'Ordre des arts et des lettres, 20 janvier 1983. 


\section{Études et carrière (1932-1984)}

\section{Études (1932-1943)}

Lycée de Saint-Etienne, 1932-1939: inscriptions au tableau d'honneur, sujets des épreuves du baccalauréat, coupures de presse relatives au baccalauréat et au concours général.

Lycée du Parc, Lyon, 1939-1945 : bulletins de notes, certificats d'immatriculation en vue de la licence d'histoire et de géographie.

\section{Carrière (1945-1981)}

Certificats et attestations de nomination dans les différents établissements scolaires :

Lyon, Lycée du Parc, maître d'internat, 1945-1946. Paris, Lycée Charlemagne, professeuradjoint d'enseignement, 1946-1950. Clermont-Ferrand, Lycée Blaise Pascal, professeur agrégé, 1950-1954. Paris, Lycée Voltaire, 1954-1957.

Paris, EPHE-VI e section / EHESS, 1957-1981: certificats de nomination de directeur d'études non cumulant, puis cumulant.

Paris, Université de Paris X-Nanterre, 1969-1977: correspondance relative à sa candidature à l'université Paris X-Nanterre; certificats de nomination; notifications d'avancement.

Göttingen, Mission historique française en Allemagne, 1977-1979: arrêté de détachement; rapport et mémoire sur la création de la Mission historique française en Allemagne.

Certificats d'exercice, rapports d'activité, listes de publications.

Missions à l'étranger, 1960-1982 : correspondance, invitations, rapports de missions.

\section{Prix et distinctions}

Annonce de la remise du prix de la fondation Thorlet de l'Académie des sciences morales et politiques, Institut de France, 27 septembre 1965.

Correspondance relative à la remise du Prix Gobert de l'Académie française, 1978: palmarès des prix littéraires, correspondance (14 lettres et cartes, 2 télégrammes).

Remise de la Médaille d'argent du CNRS, 1983 : lettres, notes et discours, photographies.

\section{Activités institutionnelles (1957-1980)}

2 Cette rubrique rassemble les documents administratifs liés à l'enseignement et aux différents organismes auxquels appartenait Robert Mandrou.

\section{Secrétariat de rédaction des annales (1954-1962)}

Robert Mandrou a été nommé secrétaire de rédaction des Annales à partir de la préparation du $\mathrm{n}^{\circ} 3$ de 1954. Le fonds comprend un dossier de correspondance spécifique aux Annales de novembre 1957 à juillet 1959. On y trouve environ 800 pelures de lettres dactylographiées signées par Robert Mandrou et approximativement autant de lettres de correspondants. Nous avons, pour cet inventaire sommaire, indiqué le nom des principaux correspondants. 
4 A cette correspondance générale relative aux Annales, s'ajoutent deux dossiers spécifiques, intitulés pour le premier : «Annales - C. R. / L. F. » qui contient un échange de lettres de Lucien Febvre et de Robert Mandrou de 1953 à 1956, dossier dans lequel R. Mandrou a ajouté par la suite un projet de réforme des Annales pour 1961. Le second dossier s'intitule « la crise - F. B. » et comprend des lettres de F. Braudel de 1960 à 1964, ainsi qu'un certain nombre de lettres adressées à R. Mandrou en 1962, à la suite de son éviction des Annales.

Annales : correspondance, novembre 1957-juillet 1959.

\begin{tabular}{|c|c|c|c|}
\hline Abrel & Bataillion. & Bemrtrand & Cahew: \\
\hline Alatonre & Elaulligg & Eibolex: & Cailloux \\
\hline Altienhein & Baumont & Bigo & Callot \\
\hline Alvenny (d') & Bayon & Eilano: & Carntimori \\
\hline Amado & Beaugrand- Blodh & Bloch & Carpentist \\
\hline Andreu & Beckesx: & Boblininskat & Castellar \\
\hline Arenilla & Benc: & Bonj.jour: & Cantillo \\
\hline Amould & Bentuassiar & Boringhinn-i & Cazenenulve \\
\hline Aron & Bequignom & Borisov & Cha:lus \\
\hline Aymard & Bera & Boulard (de) & Chappey \\
\hline Bellandiner & Bergier & Bourgint & Chatelain \\
\hline Ballarzas & Berque & Boutsuche & Chatunu \\
\hline Bauroni & Bersielt & Bowenn & Chelini \\
\hline Barthess & Berthe & Bmulez & Chevallier \\
\hline Bastide & Berti & Buckitiolz: & Chiva \\
\hline Blastie & Bertin & Cabourdin & Choieau \\
\hline
\end{tabular}


EXTRAIT DE COURS SUR “ LA FORMATION DES MYTHES NATIONAUX ".

LEÇON 37 DU 17 MARS 1971.

$$
\text { 13. II }
$$

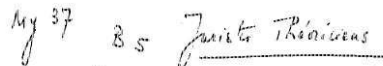

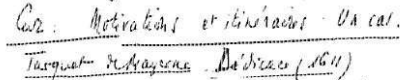

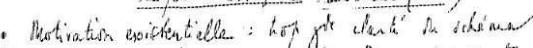

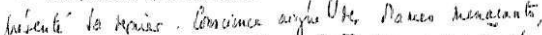

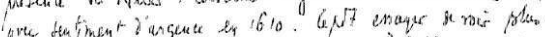

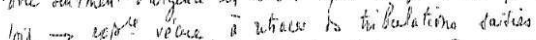

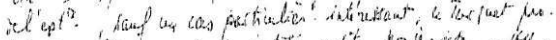

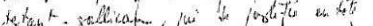

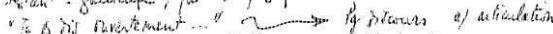

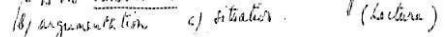

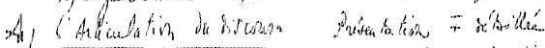

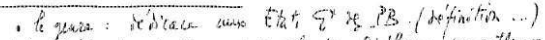

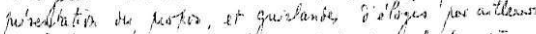

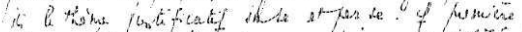

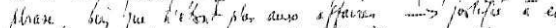

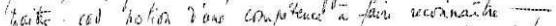

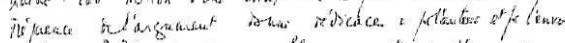

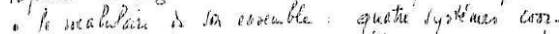

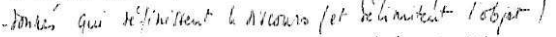

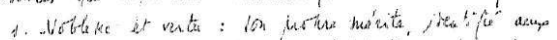

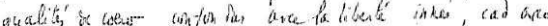

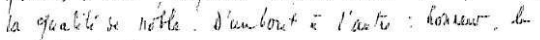
mit Let Quphing:

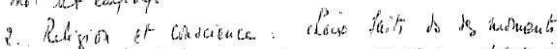

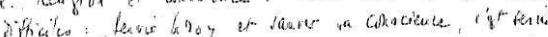

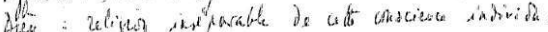
ill $\rightarrow$ ablon fistortar

\begin{tabular}{|c|c|c|c|}
\hline Selin & Tmioka & Vactert & Violunte: \\
\hline Survinki & Tspie & Yalks & Yitonet \\
\hline Setini & Tulon & Voturi & Vivanti \\
\hline Setor & Tanand & Veaurat & Wa \\
\hline Salonoua & Thourno & Vial & Wotilkats \\
\hline Sinaon & Thrume & Viallanix & wolf \\
\hline Siprial & Thuillitir & Viaire & Zagti \\
\hline Strizpok & Tomidhe & Viasoreff & Zalesdi \\
\hline Sevith & Toujat: & Viluet & Znwodati \\
\hline Spo:nar & Tourtine & Vela & Ziselo \\
\hline Stenpars & Trewor Poper & virladient & Zyproskinn \\
\hline Stranpe & Tudess & Virma & \\
\hline
\end{tabular}

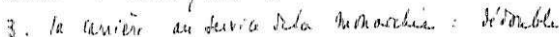
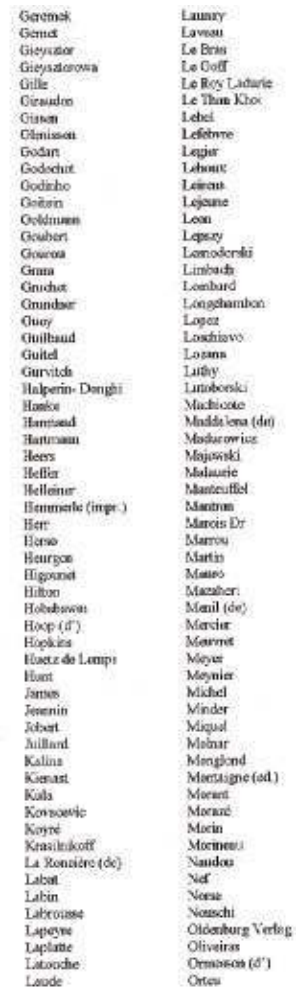

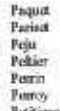

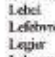

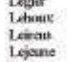

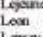
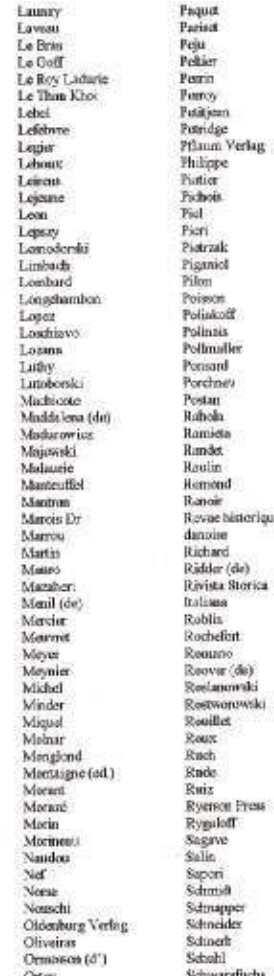

École pratique des hautes études, VIe section / École des hautes études en sciences sociales (1957-1980)

Enseignement (1957-1980)

Cahiers de présence, 1957-1980.

Dossiers de chercheurs, 1968-1976.

$\left(^{*}\right)$ lettres accompagnant le dossier 


\begin{tabular}{|c|c|c|}
\hline ADrhes, Thoous M. (*) & DYOAET, Nioole & MPAES, Albert \\
\hline ARNOLDEISAAOND & FNBGE, Ariate fenteres di & NADOU, Pime $\left({ }^{\theta}\right)$ \\
\hline Jurnes. & Funpwis Fura, Bameral & Naveut, Brigitie \\
\hline At Dotsio, Gatricl (O) & Phongeros l.suls & [Nomakiex] \\
\hline AYMONIEF, Halane Wor & Bergoren, Didier Oranumy & NoCOGULER, D. \\
\hline DUACCENL, il & 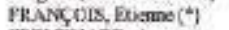 & PAKENT L LRDEUR, \\
\hline IIENרBOK, E. Maric & FRENEFHNRD, Anne & Franguies (ด) \\
\hline HERNUER, hajue & Alacie & PICARD, Frangrie- \\
\hline HERNOS, Macal (") & OABNALAT, Mato & Demingase (O) \\
\hline FOQDET. Gay & Claine $(9)$ & POLTON, Sylien \\
\hline HOUCHARD, Gisand & GUUANA, Eikabeh (") & S.ABBACH, Anthelle \\
\hline UUFLER-BILAAUD, & Cosse1.IN, Piane H (ด) & SAATSON.PIETTE, \\
\hline Rosiae 0 & ORETR, Allan (9) & Christive \\
\hline IUUROCS, Martibs & GROSPLRRIN, & SANEACON, Andol (") \\
\hline CAPTAN-FETER, & Soneviéve & SCEARTIFINNY, \\
\hline Coldete(") & HAIBIET, R- & inosigen \\
\hline CHATELUI lene (") & 1l.iolt, Atilippe & soncriteT, Dagliad \\
\hline cotrutetastruć, & KOUAL/VIALNOL!, & TANAGAW, Tobako(") \\
\hline Nonique & Catberine & TSLOAES, Marie. Therists \\
\hline CUENE, Midenlino (") & L.ASALRCIE, Bêtribe & VTCHERD, Chritine (*) \\
\hline DARMOON, Fiurns & LANCEL OT, Francine & VADID-HALEVZ, Ran (") \\
\hline TYECFE.NE-MEVILLF, & L.AKA Brow & \\
\hline lowbe & 1.1:He, Manc & Densier latinalf <ea \\
\hline DELAKERCHE⿱ & 1.KBAE TON, Jan- & attenta : \\
\hline Franpoine $(4)$ & Chinisophe $\left({ }^{\circ}\right)$ & \\
\hline IDE Vuregictor, & L.FCUIK Jem (t) & ANDRIES, Liee(") \\
\hline Annic-Therive & 1.1DENAANN & CHAPEAOT, hant (") \\
\hline DORLOT, Fresopir & MAODEL_ANNE, Midnolls & COSMOS, Thallia (") \\
\hline DUCCINL, Halane(T) & 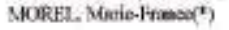 & FOSSIEAl, Frasplis [N] \\
\hline QAUTHIER, Moriqus & LECAM, Jan-Lae(") & RAMSEY, MaUthes (") \\
\hline CTLEET, Mithd (4) & OKAMOTO, Asien (") & SFNZTTTI, Franplese (") \\
\hline BAYTIC, lrmeard & PELAX, Muriv-Lsaias : & RUBIN, Elaiox (") \\
\hline JOCtraRD, hilipge & PACHaRD, Goorgea (h) & YARDENL, Nyriem \\
\hline
\end{tabular}

INSCRIPTIONS, 1965-1977.

RAPPORTS DE SOUTENANCE DE DIPLÔMES, 1960-1978.

CORRESPONDANCE ; RAPPORTS D'ACTIVITÉS ANNUELS DE L'EPHE, 1967-1973.

\section{Administration et rencontres institutionnelles (1958-1977)}

Comptes rendus de réunions; circulaires; projets d'organigramme EPHE, projets de statuts.

Rencontre interdisciplinaire, Royaumont, 1973 : convocations, notes.

Mai 1968, EPHE

Comptes rendus de réunions EPHE ; organigramme ; rapports de commissions et notes.

Mai 1968, documentation imprimée

Tracts ; revues ; réformes en cours.

Direction des collections « histoire des mentalités » (aux éd. Plon) et «10/18»(1962-1967)

5 R. Mandrou a dirigé aux éditions Plon à partir de 1962, dès sa création, la nouvelle collection « Histoire des mentalités ». Il est aussi conseiller pour la collection «10/18 ». Le fonds comprend un dossier de correspondance de 1962 à 1967. Nous donnons un inventaire des noms des correspondants comme pour les Annales. 


\begin{tabular}{|c|c|c|}
\hline A1.LPUNivD Andni & Fulske, dutile & INNDON, JinYin: \\
\hline BRLDULN, C; A. & FLOVEEST', Han & MUSCHLXCY, \\
\hline DOLUIN, Jan & FUMAZOLIL Mare & Cristiane \\
\hline FOOCDOIIE,AU, Mane & OSUZARCIUES, Jutiate & YALSSOANIIER,C. \\
\hline Cotprtasi, Colate & GOOSUL, Fnespit & MCFIEL \\
\hline CARON, Vetnat & G.JEJIN, Danit1 & MOTNOT, Clerand \\
\hline CHTTIN, Rewe & 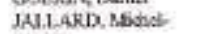 & MOGITN, EAlgar \\
\hline CIIALYilit, Lenis & Clunde & XAVALIE, Peors \\
\hline CHALMIE, Nowuiles & JOUIARD, Dhilitpe & ORY, iezanMarie \\
\hline CODNA?IDON & KIBSO, Nadis & PACLUEL, Andro \\
\hline COSTE, Nabiat & KFENGER, C. & 1ELICIER, L. \\
\hline COUULT, Not & 1.ACOMABE Maxiene & HETRE, low - Piant \\
\hline DUBY, Goregss & GAGRLANIE & PHOI IF, Adedre \\
\hline PRAT, Dyminivant & RBST, Alatre & Y, N\$L.DERBREN, Fravan \\
\hline R.ADEOWWKI & Róussin.IIE, I. A. & VERNHES, Clamile \\
\hline Anpoder(d) & RIIDE, Foumand & WARELANI, hoques \\
\hline RNDEOWWSKL G.H. (s:) & STOUFF, LNuis & \\
\hline RIBGERKOUX, Mndeleien: & THETLAY, , & \\
\hline
\end{tabular}

\section{Nanterre, université de paris X (1969-1977)}

\section{Enseignement (1969-1977)}

Fiches d'étudiants, 1971-1975.

Notes manuscrites d'étudiants pour le cours sur « La Révolution française », 1969-1970.

Administration (1972-1977)

Circulaires administratives, rapports scientifiques, brochures sur les thèmes de recherches.

Fonctionnement de l'UER de sciences juridiques : circulaires, rapports, correspondance.

\section{Göttingen, mission historique française en Allemagne (1974-1980)}

Détachement et nomination à Göttingen ; création de la Mission historique française en Allemagne (mémoire et projet) ; correspondance à propos des activités de la Mission, 1977-1980.

\section{Centre national de la recherche scientifique (1960-1973)}

Circulaires, instructions et élections.

\section{Relations franco-québécoises (1967-1973)}

\section{Enseignement au Québec (1967-1971)}

Listes d'étudiants et d'enseignants ; correspondance.

Centre franco-québécois (1972-1973)

Préparation d'un colloque, 1972 ; projet d'Institut de civilisations comparées ; projet de création d'un centre franco-québécois.

Le Conseil des arts du Canada (1968-1975)

Dossiers de demandes de subventions à la recherche (en tant que lecteur).

Laval en grève ! (1976)

Projet de convention collective entre administration et enseignants. 


\section{Unesco (1972-1976)}

Commission française pour l'Unesco, «Education » : convocations, listes des participants, projets de programme, rapports, correspondance.

Conférence de Bucarest, 21 décembre 1973: lettre de Daniel Monteux; copie de l'intervention faite au nom de la Fédération internationale syndicale de l'enseignement par Daniel Monteux.

\section{Institut national de l'audiovisuel}

Listes des productions audiovisuelles de R. Mandrou à Radio-France, 1959-1964 (données en annexe) ; correspondance de l'INA avec Christiane Mandrou, 1984-1986.

\section{Institut national de recherche et de documentation pédagogiques} (1972-1973)

Réunion sur les recherches pédagogiques en cycle primaire: "Thèmes de discussion concernant l'histoire et son enseignement à l'école élémentaire ».

Écoles normales - INRDP, 1972: projet d'un plan de travail sur l'enseignement de l'histoire à l'école élémentaire.

\section{Institut historique allemand à paris (1972)}

Notes de Robert Mandrou prises pendant un séminaire de K. F. Werner à l'Institut historique allemand ; texte de K. F. Werner, s.d.

\section{Société française d'histoire des hôpitaux (1967-1974)}

Rapports sur les mémoires reçus par la Société française d'histoire des hôpitaux pour le concours de la Société ; correspondance.

\section{Société d'histoire ecclésiastique de la France (1969)}

Projet d'enquête sur les visites pastorales : lettre-circulaire de Dominique Julia et Marc Vénard, 17.10.1969; mode d'emploi, fiche de tournée, grille codée, notice explicative, $32 \mathrm{f}$. ronéot. ; Travaux et enquêtes pour un répertoire des visites pastorales, Revue d'histoire de l'Eglise de France, tiré à part, s.d.

\section{Réformes de l'enseignement (1974-1975)}

\section{Réforme Fontanet [1974]}

Article de Robert Mandrou pour Le Monde et correspondance.

Réforme Haby (1975-1977)

Projet d'article pour Le Monde (texte refusé) ; articles sur la réforme ; correspondance. 


\section{Activités d'enseignement et de recherche}

Cette rubrique rassemble les manuscrits et les documents préparatoires des deux thèses, les notes des cours et des conférences, les notes de lecture et les fichiers de travail.

\section{Thèses d'état (1958-1968)}

Thèse principale: Magistrats et sorciers en France au $\mathrm{XVII}^{\mathrm{e}}$ siècle: une analyse de psychologie historique.

Exemplaire manuscrit, $611 \mathrm{f}$. ; documents préparatoires pour la thèse.

Thèse complémentaire : Les Fugger, propriétaires fonciers en Souabe (1560-1618) : étude de comportements socio-économiques à la fin du $\mathrm{XVI}^{\mathrm{e}}$ siècle.

Exemplaire manuscrit, $171 \mathrm{f}$; ; exemplaire dactylographié, $299 \mathrm{f}$.

Notices d'information; circulaire ; inscriptions, 1959; convocation pour la soutenance, 1968 ; notes de travail.

\section{Cours (1948-1977)}

Nous avons respecté les titres de R. Mandrou pour les notations typographiques des siècles $\left(\mathrm{xvI}^{\mathrm{e}}\right.$ ou $\left.16^{\mathrm{e}}\right)$.

\section{Leçons de l'enseignement secondaire}

EPHE-VI'e section / EHESS (1957-1977)

1957-1958 : «Expansion française-XvII ", notes de cours, 49 f. ms.

1960-1961, 1961-1962, 1962-1963: " Recherches sur la littérature populaire des XVII ${ }^{\mathrm{e}}$ et XVIII ${ }^{\mathrm{e}}$ siècles ", notes de cours, 18 leçons, 274 p. ms.

1963-1964, 1964-1965, 1965-1966: "Economies et mentalités rurales: l'exemple de la Souabe bavaroise à la fin du XvI $\mathrm{e}^{\mathrm{e}}$ siècle », notes de cours, 34 leçons, $169 \mathrm{p}$. ms.

1965-1966 : plan du cours sur l'endettement paysan et programme général de l'année.

1966-1967: «Recherche sur les comportements séditieux dans la France du XVII ${ }^{e}$ siècle », notes de cours, 15 leçons, 62 p. ms.

1967-1968: « La sorcière de village ", notes de cours, 21 leçons, 82 p. ms.

1968-1969, 1969-1970, 1970-1971, 1971-1972, 1972-1973 : « Recherches sur la formation des mythes nationaux en France, $\mathrm{XVI}^{\mathrm{e}}$-XVII ${ }^{\mathrm{e}}$ siècle », cours en quatre parties, notes de cours, 86 leçons, 369 p. ms.

1973-1974, 1974-1975, 1975-1976: «Recherches sur les échanges intellectuels entre France et Allemagne au XVII e siècle : le cas du duc August à Wolfenbüttel (1635-1666)», notes de cours, 43 leçons, 172 p. ms.

1976-1977 : « Débats et combats. Textes inédits : possessions et sorcellerie au XVII ${ }^{\mathrm{e}}$ siècle », notes de cours, 19 leçons, 70 p. ms.

Sessions de fin d'année : séminaires bilans (1968-1977)

11-14 septembre 1968, Cerisy-la-Salle

«Mai-juin 1968 », liste des participants ; plan, $1 \mathrm{f}$. ms. ; programme sur trois jours, $1 \mathrm{f}$. ms. ; notes de travail, $3 \mathrm{f}$. ms. ; notes, $6 \mathrm{f}$. ms. ; correspondance.

5-8 juillet 1969, Cerisy-la-Salle 
«Echanges de vue sur les méthodes en histoire », notes, 9 p. ms.

25-27 juin 1974, Azay-le-Ferron

«Discussion et réflexion sur la culture contemporaine », notes, $23 \mathrm{f}$. ms.

24-26 juin 1975, Azay-le-Ferron

«Les méthodes de l'histoire des mentalités à travers la production récente ", notes, $14 \mathrm{f}$.

ms.

22-24 juin 1976, Troyes

Notes, 14 f. ms.

19-22 juin 1977, Troyes

Circulaire.

\section{Paris X-Nanterre (1969-1976)}

1968-1969: "Nouveaux intellectuels dans la société occidentale européenne: des humanistes aux libertins érudits» [sur la couverture de la chemise: Place des intellectuels XVI ${ }^{\mathrm{e}}-\mathrm{XVII} \mathrm{e}^{\mathrm{e}}$, notes de cours, 22 leçons, 44 p. ms.

1968-1969: « Les révoltes populaires au XVII ${ }^{\mathrm{e}}$ siècle », notes de cours, 14 f. ms.

1968-1969 : Cours d'agrégation. «La société française de 1680 à 1789 : structures sociales, culture, modes de vie » [sur la couverture de la chemise: Cultures], notes de cours, 10 leçons, 40 p. ms.

1969-1970 : «L'Ancien Régime et la Révolution française », notes de cours, 28 leçons, 56 p. ms.

1969-1970 : «Jansénisme(s) en France aux 17 $17^{\mathrm{e}} 18^{\mathrm{e}}$ siècles », notes de cours, 12 f. ms. pour la première partie; $21 \mathrm{p}$. ms. pour la seconde partie.

1970-1971 : « Méthodologie », notes de cours, 24 leçons, 48 p. ms.

1970-1971 : «Livre et société en France au XVII siècle », notes de cours, 30 f. ms.

1970-1971: Cours d'agrégation. «Les Iles britanniques (1630-1714)», notes de cours, 19 leçons, 46 p. ms.

1971-1972 : « Pays-Bas et Provinces unies », notes de cours, 14 leçons, 54 p. ms.

1971-1972 : « Ethiques religieuses et esprit du capitalisme », notes de cours, $36 \mathrm{f}$. ms.

1971-1972: Cours d'agrégation. «Les Iles britanniques (1630-1714)» (suite), notes de cours, 6 leçons, 19 p. ms.

1972-1973 : «Genève », notes de cours, 21 leçons, 42 p. ms.

1972-1973 : «Vénalité des charges et recrutement du personnel monarchique en France au XVII ${ }^{\mathrm{e}}$ siècle ", notes de cours, $31 \mathrm{f}$. ms.

1972-1973 : Cours d'agrégation. "Charles Quint : monarchie universelle et nationalisme », notes de cours, 18 leçons, 44 p. ms.

1973-1974 : «Rationalité politique et société en Europe au 18 siècle (1715-1760) » [sur la chemise «Raison d'État et sociétés en Europe (1715-1780) »], notes de cours, 21 leçons, 40 f. ms.

1973-1974 : « La vie urbaine au XVIII siècle : Lyon », notes de cours, 28 f. ms.

1973-1974 : Cours d'agrégation. «La vie urbaine en Allemagne au temps de Charles Quint (1516-1555) » [sur la chemise «Charles Quint: la vie urbaine dans le SERG $1^{\text {re }}$ moitié du $\left.\mathrm{XVI}^{\mathrm{e}} »\right]$, notes de cours, 16 leçons, 40 p. ms.

1974-1975 : « Méthodologie I », notes de cours, 25 leçons, 54 p. ms. ; bibliographie, 3 f. ms. 1974-1975: « Négociants et fermiers généraux au XviII ${ }^{e}$ siècle », notes de cours, 17 f. ms.

1974-1975: Cours d'agrégation. "La famille, l'enfant et l'éducation en France et en Grande-Bretagne du XVI ${ }^{e}$ au XVIII siècle ", notes de cours, 21 leçons, 44 p. ms. 
1975-1976: «Réformes et réformés en France du XVI ${ }^{\mathrm{e}}$ siècle jusqu'aux guerres de religion ", notes de cours, 14 leçons (manque la leçon 12), 25 p. ms. (cours incomplet se terminant le 4 mars 1976).

1975-1976: Cours de licence (enseignement spécialisé). "Vie rurale aux $\mathrm{XVI}^{\mathrm{e}}$ et $\mathrm{XVII}{ }^{\mathrm{e}}$ siècles ", notes de cours, 14 leçons, $25 \mathrm{f}$. ms. (cours incomplet se terminant le 4 mars 1976).

1975-1976 : « Méthodologie II », notes des leçons faites par R. Mandrou (1, 2, 3, 8, 9, 10, 13), 14 f. ms.

1976-1977 : «L'Autriche et le monde danubien (1683-1780) », notes de cours, 26 leçons, 53 p. ms.

1976-1977 : Cours de licence (enseignement spécialisé). « [Les révoltes populaires au XVII ${ }^{\mathrm{e}}$ siècle] », d'après Y. M. Bercé, notes de cours, 23 f. ms.

1976-1977 : Cours d'agrégation. « La Nouvelle-France » [sur la couverture de la chemise], notes de cours, 21 leçons, 42 p. ms.

\section{Séminaires, Canada (1963-1975)}

1963, Québec, Université Laval :

«Le $1^{\mathrm{er}}$ jansénisme français : 1640-1668 », plan en 15 leçons, $1 \mathrm{f}$. ms. ; 10 questions pour exposés d'étudiants, 2 f. ms. ; notes de cours, 15 leçons, 60 p. ms. ; 17 fiches ms.

1965, Québec, Université Laval :

«Mentalités des milieux coloniaux au XVII ${ }^{\mathrm{e}}$ siècle», notes de cours reprises d'une conférence pour l'Ecole normale supérieure, "L'expansion française hors d'Europe au XVII ${ }^{\mathrm{e}}$ siècle », s.d., 6 f. ms. ; notes de cours reprises du cours de l'EPHE (1957-1958), « Recherche sur l'expansion des français hors d'Europe au XVII ${ }^{\mathrm{e}}$ siècle », $6 \mathrm{f}$. ms. ; notes de travail, 16 f. ms.

1967, Québec, Université Laval :

- «Attitudes séditieuses dans la société française du XVII siècle ", plan en 25 leçons (sur la chemise); notes de cours, 25 leçons, $26 \mathrm{f}$. ms.; plan de «Vingt ans après ou bilan, direction de recherches, 1948-1968 », 1 f. ms.

- «Europe moderne », notes de cours, 16 leçons, 16 f. ms.

- Conférences :

"Culture populaire en France aux $\mathrm{XVII}^{\mathrm{e}}-\mathrm{XVIII}{ }^{\mathrm{e}}$ siècles: regard ethnologique sur une culture oubliée ", "Le Pèlerin perdu, film ethno-historique ", "Sensibilité religieuse et religion vécue en France au XVII ${ }^{\mathrm{e}}$ siècle : le sentiment de la peur » (conférences faites à Ottawa), notes de travail, 7 f. ms.

1969, Québec, Université Laval :

- "Etudes de documents inédits sur les procès de sorcellerie au XVII ${ }^{\mathrm{e}}$ siècle ", programme, 2 f. ms. ; 7 textes documentaires, 7 f. ronéot. avec annotations ms.

- Conférences :

"Mentalités en France et Nouvelle-France au XVII ${ }^{\mathrm{e}}$ siècle : programme de recherches ", notes de conférence, 2 p. ms. ; lettre de Yves de Zoltvany, Mc Gill University, Montréal, 29.9.1969, lettre de Laurier L. Lapierre, 10.10.1969.

« Mentalité religieuse et moralité (1630-1660)», notes de conférence, 3 p. ms. ; circulaire de l'université d'Ottawa pour le 14 et 21.10.[1969].

"Métier d'historien aujourd'hui », notes de conférence, 2 p. ms. ; lettre de Maurice Carrier, Université du Québec, Trois Rivières, 25.9.1969. 
"Problèmes universitaires français : rentrée 1969 », notes de conférence, Université du Québec, Trois Rivières, 2 p. ms.

"La Révolution française et l'histoire du Québec », notes de conférence, 2 p. ms.

«Politique et historiographie », notes de conférence, $1 \mathrm{f}$. ms.

"Sorcellerie et possession en France au $\mathrm{XVII}^{\mathrm{e}}$ : problème de mentalité religieuse », notes de conférence, $1 \mathrm{f}$. ms.

"Mentalités religieuses dans la France de l'ouest dans la seconde moitié du XVII ${ }^{\mathrm{e}}$ siècle ", notes de conférence, 2 p. ms.

«Libertinage érudit, vie intellectuelle/scientifique et nouvelle définition de la morale (sociale) », notes de conférence, $1 \mathrm{f}$. ms.

Brouillon manuscrit d'une lettre de recommandation pour Fernand Ouellet.

1971, Canada, Université d'Ottawa :

- "Intellectuels et vie intellectuelle en France au XVI ${ }^{e}$ siècle ", plan en 12 leçons, 1 f. ms. ; plan de J. H. Plumb adapté, 1 f. ms. ; notes de cours, 12 leçons, 12 f. ms.

- " Médecine et possessions diaboliques en France au XVII ${ }^{e}$ siècle », plan en 24 leçons, $1 \mathrm{f}$. ms. ; programme, $1 \mathrm{f}$. ms. ; notes de cours, 24 leçons, $23 \mathrm{f}$. ms. ; textes documentaires, $11 \mathrm{f}$. ms.

- "Métier d'historien / d'enseignant d'histoire ", plan en 12 leçons, 1 f. ms. ; notes de cours, 12 f. ms. ; calendrier pour les séminaires d'Ottawa, 1971, 3 f. ms.

- Conférences extérieures :

Notes pour 6 conférences, $6 \mathrm{f}$. ms. : « Mouvement dans l'histoire économique actuelle en France »; «Les Annales et l'historiographie française, 1929-1956-1971»; "Courants actuels de l'historiographie française »; "Le Grand siècle, une révision »; "Idée de monarchie en France de l'Ancien Régime / XVII e siècle »; «La sorcellerie en France sous l'Ancien Régime »; notes de travail, 6 f. ms.

1973, Québec, Université Laval :

- "Les révoltes populaires en France dans la première moitié du XvII siècle", présentation, plan et bibliographie, 2 p. ms., 1 f. ronéot.; notes de cours, 16 p. ms. ; $13 \mathrm{f}$. de textes documentaires à reproduire, annotations ms.

- Cours II : [« Formation du sentiment national, $\mathrm{XVI}^{\mathrm{e}}-\mathrm{XVII}^{\mathrm{e}}$ siècle »], plan du séminaire, liste des étudiants, 2 p. ms.; notes de cours, 9 leçons, 17 p. ms., $7 \mathrm{f}$. ms. de textes documentaires, 10 fiches ms.

1975, Canada, Université d'Ottawa :

- "Louis XIV », plan en 25 leçons, 2 f. ms. ; notes de cours, 23 leçons, 46 p. ms., textes documentaires, 9 f. ronéot., bibliographie, $1 \mathrm{f}$. dactyl. ; textes documentaires, $12 \mathrm{f}$. impr.

- « Histoire sociale et histoire des mentalités ", plan en 7 leçons, $1 \mathrm{f}$. ms. ; notes de cours, 5 f. ms.

[ "L'enseignement dans la France des XVII ${ }^{e}-\mathrm{XVIII}^{\mathrm{e}}$ siècles »], notes de travail, 7 f. ms.

- Conférences :

« Magistrats et sorciers en France au XVII ${ }^{e}$ siècle » (conférence), notes de travail, 3 f. ms.

"Les mentalités populaires aux XVII ${ }^{\mathrm{e}}$ et XVIII ${ }^{\mathrm{e}}$ siècles », « Mentalités collectives et histoire sociale », "Economie et société française $-\mathrm{XVI}^{\mathrm{e}}-\mathrm{xVIII}{ }^{\mathrm{e}}$ siècle : tendances de recherche », "La France moderne $\mathrm{XVI}^{\mathrm{e}}$-XVIII ${ }^{\mathrm{e}}$ siècle : les cultures françaises", "Vie intellectuelle en Europe $\mathrm{XVI}^{\mathrm{e}}-\mathrm{XVII}^{\mathrm{e}}$ siècle ", "Magistrats et sorciers en France au XVII siècle ", plans de travail, 10 p. ms.

" Recherches en cours ", « Histoire et historien », « Louis XIV en son temps ", « Recherche sur la vie culturelle en France et en Angleterre (1660-1700) », plans de travail, 6 p. ms. 
«Littérature et histoire : France des XVII ${ }^{\mathrm{e}}$ et XVIII ${ }^{\mathrm{e}}$ siècles ", «La place de l'histoire de la civilisation dans l'enseignement en France ", " La vie intellectuelle en France au XVIIe », "Louis XIV en son temps : une révision historiographique ", «Introduction à l'histoire de la civilisation française ", plans de travail, $5 \mathrm{f}$. ms.

"Culture citadine en France aux XVII ${ }^{\mathrm{e}}$ et XVIII ${ }^{\mathrm{e}}$ siècles ", " La vie intellectuelle en France et en Europe au début du XVII ${ }^{\mathrm{e}}$ siècle ", plans de travail, $2 \mathrm{f}$. ms. ; "Cultures populaires et savantes : rapports et contacts » (communication), notes de travail, $10 \mathrm{f} . \mathrm{ms}$.

\section{Séminaires, Paris, Université de Paris VII, UER de géographie et sciences de la société (1970-1972)}

1970-1971 : "L'histoire au Xx siècle », programme, 1 f. ronéot., notes de cours, 3 séances, $3 \mathrm{f}$. ms. ; programme des séances de travaux pratiques, $2 \mathrm{f}$. ronéot.

1970-1971 : « Historiographie », notes de travail, 16 p. ms., 6 f. ronéot.

1971-1972 : «Historiographie », 2 lettres-circulaire, 9.11.1971, 22.11.1971; programme, 1 f. ms. (à l'intérieur de la chemise et cachant le programme de 1970-1971, 1 f. ms.) ; notes de travail, 20 p. ms.

\section{Séminaires, Paris, Université de Paris X-Nanterre, UER de lettres anciennes et modernes et de linguistique, DEA (1975-1978)}

1976-1977 : « Littérature et mentalités, $17^{\mathrm{e}}$ et $18^{\mathrm{e}}$ siècles »; « Libertins érudits : le cas de N. Peiresc », 2 p. ms. ; « Nicolas Peiresc (1580-1637) », 2 p. ms. ; « Peiresc, le savant », 2 p. ms. Chemise « DEA Zuber » avec le plan de cinq séminaires, [1975-1976 ?].

Lettre de M. Zuber pour le séminaire 1978-1979, 25.4.1978.

\section{Enquête sur Plozévet (finistère) (1961-1971)}

Préparation et déroulement de l'enquête pluridisciplinaire «Plozévet (Finistère)»: rapports annuels d'activité ; notes manuscrites de R. Mandrou pour un article ; résumés dactylographiés de l'article sur l'enquête de Plozévet ; placards d'un article pour la Revue historique; correspondance.

Rapports de fin d'enquête pour la Délégation générale à la recherche scientifique (DGRST) ; pré-rapport de synthèse par André Burguière, $171 \mathrm{p}$.

\section{Notes de lecture et fichiers}

\section{Notes de lecture}

7 Ces notes manuscrites ou dactylographiées en vue d'un compte rendu de lecture étaient placées dans les ouvrages de la bibliothèque de R. Mandrou. Elles sont au nombre de 320 feuillets. Elles ont été répertoriées par ordre alphabétique des auteurs; la liste des ouvrages est en annexe.

\section{Fichiers}

Ces fichiers de travail, constitués par R. Mandrou, se présentaient dans une dizaine de boîtes identiques, fabriquées par lui-même. Selon leur contenu, nous avons donné un intitulé général (France, Bibliothèque bleue, etc.). Pour la France, les rubriques numérotées 
par R. Mandrou correspondent au plan, placé en tête du fichier, et aux onglets. Cet ensemble de fiches est organisé en entrées thématiques.

France

1 - Tempérament ; 2 - Vie affective : 1 fichier

3 - Vie morale ; 7 - Vie économique ; 8 - Vie politique : 1 fichier

4 - Vie intellectuelle : 2 fichiers

5 - Religion : 2 fichiers

6 - Vie sociale : 1 fichier

Bibliothèque bleue : 1 fichier

Sorcellerie : 2 fichiers

Fugger : 4 fichiers

Mythes nationaux : 1 fichier

Wicquefort : 1 fichier

\section{Communications scientifiques (1958-1977)}

Cet ensemble de documents, constitué par des textes de communications et des notes prises lors de colloques et conférences, est présenté par ordre chronologique et semble être à peu près exhaustif, sauf pour la période 1972-1973 pour laquelle il manque, en particulier, les textes des conférences faites à l'étranger (Japon et Israël).

\section{Missions d'études, colloques et conférences (1958-1977)}

Allemagne de l'Ouest, 1958-1962, missions d'études: rapports de missions, 1958, "Recherches sur l'histoire intellectuelle et économique allemande du XVII ${ }^{\mathrm{e}}$ siècle », $9 \mathrm{f}$. copie dactyl.; 1959, "Recherches sur les comportements et mentalités des familles marchandes d'Augsbourg (1570-1630)», $8 \mathrm{f}$. copie dactyl.; 1960, "Recherches sur les comportements et mentalités des familles marchandes d'Augsbourg (1570-1630)», $6 \mathrm{f}$. copie dactyl.; 1961, "Recherches sur les comportements et mentalités des familles marchandes d'Augsbourg (1570-1620)», $5 \mathrm{f}$. copie dactyl.; 1962, "Recherches sur les comportements et mentalités des familles marchandes d'Augsbourg (1570-1620)», $5 \mathrm{f}$. copie dactyl. ; notes de conclusion, $10 \mathrm{f}$. ms.

Aix-en-Provence, 18-19 juin 1959, conférences : «Introduction à la recherche sur les mentalités", notes de conférence, $6 \mathrm{f}$. ms.; notes, $3 \mathrm{p}$. ms.; graphique, $1 \mathrm{f}$. ronéot.; «Expansion coloniale française et hollandaise au XVII siècle", notes de conférence, $5 \mathrm{f}$. ms.

Lyon, 14 décembre 1961, conférence, [Union rationaliste] : «Aux origines du renouveau catholique français du $\mathrm{Xx}^{\mathrm{e}}$ siècle ", notes de conférence, $5 \mathrm{f}$. ms.

Aix-en-Provence, 29 août-4 septembre 1962, Second International Economic History Conference : « Le Concept de classe ", notes de conférence, 6 f. ms.

Pise, 4-7 mai 1962, conférences, Faculté des lettres et de philosophie: "Sociologie historique de la pratique religieuse (catholique) en France au XvII siècle", notes de conférence, $7 \mathrm{f}$. ms. ; « Progrès de la pratique religieuse au cours du XVII ${ }^{e}$ siècle ", notes de conférence, $5 \mathrm{f}$. ms. ; « Pratique et vitalité religieuse (catholique) au XVII ${ }^{\mathrm{e}}$ siècle », notes de conférence, 7 f. ms. ; « La pratique religieuse catholique en France au XVII siècle : bilan de recherches et positions de problèmes ", article reprenant l'essentiel des trois conférences de Pise, 1963, 22 f. dactyl. ; " La pratique religieuse catholique en France au XVII ${ }^{e}$ siècle ", 
article résumant une conférence faite à l'université de Lublin le 2 mai 1963, 7 f. copie dactyl.; notes de travail sur « Les jésuites au XVII ${ }^{\mathrm{e}}$ siècle », $4 \mathrm{f}$. ms.

Saint-Etienne, 19 janvier 1963, conférence : « Métier d'historien aujourd'hui », notes de conférence, $6 \mathrm{f}$. $\mathrm{ms}$.

Pologne (Wroclaw, Cracovie, Varsovie, Lodz, Lublin), 20 avril-8 mai 1963, conférences : programme de la mission, $2 \mathrm{f}$. dactyl. ; 2 rapports de mission, $5 \mathrm{f}$. copie dactyl. (1 pelure, $4 \mathrm{f}$. dactyl.) ; textes des conférences : «Culture populaire et culture savante dans la France d'Ancien Régime », notes, 2 p. ms. ; "Culture savante et culture populaire dans la France des $\mathrm{XVII}^{\mathrm{e}}$ et $\mathrm{XVIII}^{\mathrm{e}}$ siècles: la littérature de colportage », $15 \mathrm{f}$. copie dactyl. ; "Les recherches actuelles sur la civilisation française du XVII ${ }^{\mathrm{e}}$ siècle ", notes, $4 \mathrm{f}$. ms.; "Recherches actuelles sur la civilisation française du XVII ${ }^{\mathrm{e}}$ siècle », $6 \mathrm{f}$. copie dactyl.; « [Nouvelles] perspectives sur la civilisation française du $17^{\mathrm{e}}$ siècle », 2 f. ms. ; " Sociologie religieuse historique régressive : un exemple de collaboration, ou de contact ", notes, $3 \mathrm{f}$. ms. ; "La pratique religieuse catholique en France au XVII ${ }^{\mathrm{e}}$ siècle ", $7 \mathrm{f}$. copie dactyl. ; "Objectifs et méthode d'une histoire des civilisations", notes, $5 \mathrm{f}$. ms. ; "Objet et méthodes d'une histoire des mentalités ", notes, $6 \mathrm{f}$. ms.

Pays-Bas (Utrecht, Groningue), 16-22 mars 1964, conférences : "Situation de Lucien Febvre dans l'historiographie française ", « Pensée de Lucien Febvre », notes, 6 f. ms.

Monaco, 21-26 mai 1965, colloque "Sciences humaines quantitatives et culture », Centre international d'étude des problèmes humains : " Du quantitatif en histoire ", notes pour l'exposé, 4 f. ms.

Munich et Vienne, 23 août-3 septembre 1965, colloques: programme du troisième Congrès international d'histoire économique (Munich), 23-27 août 1965, 2 p. impr. ; texte non rédigé « Ravitaillement de l'Assistance publique, 1820-1870 : la ration de restauration à Paris au XIX siècle ", $8 \mathrm{f}$. ms.; résumé de la communication, $1 \mathrm{f}$. copie dactyl.; communication « Ravitaillement de l'Assistance publique à Paris au XIX siècle ", $6 \mathrm{f}$. copie dactyl.; 1 autre exemplaire avec bibliographie, $15 \mathrm{f}$. copie dactyl.; 1 exemplaire pour publication pour Jahrbücher für Nationalökonomie und Statistik, $1^{\text {res }}$ épreuves impr.; texte d'une communication « Un exemple de définition : un concept de classe », $11 \mathrm{f}$. dactyl. ; 1 copie dactyl.

Etats-Unis, Ann Arbor, $1^{\text {er }}$ avril 1966, conférence, Society for French Historical Studies : Program for the Twelfth Annual Conference of the Society for French Historical Studies, $1 \mathrm{f}$. ronéot. ; «L'historiographie française des XVI ${ }^{\mathrm{e}}-\mathrm{XVIII}$ [ [siècles] : bilans et perspectives ", $8 \mathrm{f}$. ms., exemplaire ronéot., $5 \mathrm{f}$.

Genève, 5-6 mai 1967, deuxième colloque franco-suisse d'histoire économique : programme.

Tours, 4-9 avril 1968, 93 congrès national des sociétés savantes: notes pour sa communication «Un problème de diététique à l'Hôtel Dieu de Paris à la veille de la Révolution », 8 f. ms., 53 fiches ms. ; résumé de la communication de Munich, 1 f. copie dactyl. ; « Le Ravitaillement d'une ville dans la ville : la ration alimentaire de restauration à l'Assistance publique à Paris (1820-1870) », extrait de L'Hôpital et l'aide sociale à Paris, $\mathrm{n}^{\circ}$ 43, janv.-févr. 1967, p. 2-11.

Budapest, 15-21 mai 1968, conférences, Université Lorand Eötvös : correspondance.

Strasbourg, 1969 : préparation du colloque « Strasbourg, au cœur religieux du XVI ${ }^{\mathrm{e}}$ siècle : mentalités et sociétés ", [initialement prévu pour avril 1969], notes, 3 f. ms.

Reims, avril 1970, 95 congrès national des Sociétés savantes, section d'histoire moderne et contemporaine : programme régional. 
Pologne (Varsovie, Lublin, Torun, Cracovie), 11-20 octobre 1970, conférences: "Sorcellerie rurale et chasse aux sorcières dans la France du XVII ${ }^{\mathrm{e}}$ siècle ", notes de conférence, $1 \mathrm{f}$. ms.; "Les soulèvements populaires ", notes de conférence, $2 \mathrm{p}$. ms. ; «Réflexions historiennes sur mai 1968 », notes de conférence, 1 f. ms. ; « Sorcellerie et histoire sociale ", notes de conférence, $1 \mathrm{f}$. ms.

Paris, avril 1971, conférence, École des Chartes : notes sur « Histoire culturelle et histoire des mentalités », 2 p. ms.

Budapest, 16-22 mars 1972, colloque "Paysannerie française, paysannerie hongroise »: notes prises lors du colloque, $16 \mathrm{f}$. ms.; programme du colloque de septembre « Mouvements paysans en Europe ", $1 \mathrm{f}$. ms.

Aix-en-Provence, 22-24 septembre 1972, colloque «Historiographie de la Réforme»: notes pour sa communication «Lucien Febvre et la réforme ", $7 \mathrm{f}$. ms.; notes pour le "Rapport sur le renouvellement historiographique», $4 \mathrm{f}$. ms.; photocopies de textes dactylographiés pour le colloque: R. Mandrou... [et al.]; notes prises lors des communications, 3 f. ms.

Ratisbonne, 2-5 octobre 1972, congrès des historiens allemands : plan du rapport de R. Mandrou, 2 f. ms. ; notes pour le rapport, 9 f. ms. ; rapport, 14 f. ms.

Paris X-Nanterre, 15 juin 1973, colloque sur la place de l'histoire dans les filières nouvelles : compte rendu du colloque, 5 p. ronéot. ; rapport du département d'histoire de la faculté de Paris X-Nanterre, 4 p. ronéot. ; notes de la réunion de la commission de la faculté de Paris X-Nanterre du 16.1.[1973], $1 \mathrm{f}$. ms.; notes de réunion, $1 \mathrm{f}$. ms. ; notes prises lors du colloque, $7 \mathrm{f}$. ms. ; notes sur l'enseignement en maîtrise, $6 \mathrm{f}$. ms.

Besançon, 25-29 mars 1974, $99^{\mathrm{e}}$ congrès national des sociétés savantes, section d'histoire moderne et contemporaine : programme régional.

Villetaneuse, 17 mai 1974, journée de formation permanente, « Réflexion sur les contenus de l'enseignement de l'histoire », Université de Paris-Nord, UER de lettres et sciences humaines : plan de l'exposé, $1 \mathrm{f}$. ms. ; notes pour l'exposé, $3 \mathrm{f}$. ms.

Wolfenbüttel, 27 septembre- $1^{\mathrm{er}}$ octobre $1974,12^{\mathrm{e}}$ colloque historique franco-allemand de l'[Institut historique allemand] Paris : notes prises lors des communications, 15 p. ms. ; notes, $3 \mathrm{f}$. ms. ; communication de R. Mandrou, « La méthode historique de Voltaire : une lecture du siècle de Louis XIV » : texte de l'article, $12 \mathrm{f}$. ms., exemplaire copie dactyl., $15 \mathrm{f}$. ; notes de la communication, 7 f. ms., 12 fiches ms. ; 17 fiches ms.

Paris, 4 mars 1975, conférence, Ecole nationale supérieure des Mines : notes pour l'exposé " Histoire quantitative élémentaire des comportements », $3 \mathrm{f}$. ms.

Stanford, 20-25 avril 1975, symposium on «Popular Culture and Learned Culture in France : the Seventeenth to the Twentieth Centuries », University: "Cultures populaire et savante : rapports et contacts », $24 \mathrm{f}$. ms. ; 1 exemplaire, $35 \mathrm{f}$. dactyl. ; plan de " Culture populaire et culture savante dans la France d'Ancien Régime », $1 \mathrm{f}$. ms. ; plan de «Culture populaire et culture savante : rapports, contacts et originalités ", $1 \mathrm{f}$. ms.

Göttingen, 20-24 mai 1975, colloque international «Probleme des Übergangs vom Ancien Régime zur Révolution in Frankreich», Max-Planck-Institut für Geschichte : communication "Culture populaire et mouvements populaires dans la transition de l'Ancien Régime à la Révolution », 19 f. ms., 1 exemplaire ronéot., 15 f., 2 cartes.

Strasbourg, 25-29 mai 1975, colloque international «Strasbourg au cœur religieux du XVI ${ }^{\mathrm{e}}$ siècle ", hommage à Lucien Febvre, Université : programme, 8 p. impr.; notes en allemand, $6 \mathrm{f}$. ms. ; notes prises lors du colloque, $3 \mathrm{f}$. ms. ; notes sur « Courants actuels de l'historiographie française », $1 \mathrm{f}$. ms. 
Mulhouse, 5 décembre 1975, conférence : notes pour la conférence «Les faux sorciers début XVIII ${ }^{\mathrm{e}}$ siècle ", $5 \mathrm{f}$. ms.

Colmar, 6 décembre 1975, conférence : notes pour la conférence « Les révoltes paysannes en France au XVII ${ }^{\mathrm{e}}$ siècle ", $2 \mathrm{f}$. ms.

Londres, 8-9 mars 1976 [ou 24-25 mai ?], conférences : notes pour ses conférences, « Les hommes de science dans la seconde moitié $d u x^{2}{ }^{e}$ ", "De la culture populaire en France ", $5 \mathrm{f}$. ms.

Louvain-la-Neuve, 31 mai-3 juin 1976, séminaire professionnel, Organisation de coopération et de développement économiques (OCDE), Centre pour la recherche et l'innovation dans l'enseignement: programme sur la gestion des établissements d'enseignement supérieur : la gestion de la recherche universitaire.

Paris, 8-11 juin 1976, $\mathrm{II}^{\mathrm{e}}$ réunion du groupe de consultants sur «Les finalités et les théories de l'éducation », Organisation des nations unies pour l'éducation, la science et la culture (UNESCO), organisée conjointement par la division de la philosophie et le bureau international d'éducation : notes manuscrites de R. Mandrou, $7 \mathrm{f}$.

Troyes, 11-13 octobre 1976, colloque franco-hongrois sur François II Rákóczi : notes prises lors du colloque, $9 \mathrm{f}$. ms. ; notes pour son exposé « Les écrits mystiques de Rákóczi », $3 \mathrm{f}$. ms.; notes et correspondance pour son article sur les «Mémoires et confessions du prince François II Rákóczi, textes choisis par Béla Kópeczi », 11 f. ms., 4 lettres dactyl.

Privas, mai 1977, colloque « Histoire et clandestinité du Moyen Age à la Première guerre mondiale » : souscription, 3 p. impr.

Marseille, 28-30 juillet 1977, septième colloque de Marseille sur le XvII ${ }^{\mathrm{e}}$ siècle «La Qualité de la vie au XVII siècle", Centre méridional de rencontres sur le $\mathrm{XVII}^{\mathrm{e}}$ siècle : notes manuscrites de R. Mandrou.

Francfort-sur-le-Main, 18-19 mai 1979, 6. Römerberggespräche «Die Angst des Prometheus. Fortschritt ohne Sinn ? » : notes de R. Mandrou, 1 f. ms.

\section{Notes et textes de conférences}

Nous avons regroupé ici les textes des conférences dont la date ou le lieu n'ont pas été identifiés.

"Place d'une histoire des mentalités dans l'enseignement secondaire»: notes de conférence, s.d., 2 f. ms.

«Structures et conjonctures dans l'économie française d'Ancien Régime»: notes de conférence, s.d., 6 f. ms.

"La méthode historique aujourd'hui »: séminaires, Centre d'études sociologiques (J. Dumazedier), 21.12.[1962]-11.1.[1963], notes des conférences, 12 f. ms. ; bibliographie, 2 f. ronéot.

"Problèmes et méthode de l'historiographie française d'aujourd'hui »: conférence, [Canada], notes de conférence, s.d., $1 \mathrm{f}$.

« Le vocabulaire social et culturel » : texte ronéot., s.d., 10 p.

«La communauté rurale: problème d'équilibre de la micro-société d'Ancien Régime : l'exemple de Sennely-en-Sologne » : notes de conférence, s.d, 1 f. ms.

« Modèles culturels de la France moderne/urbaine » : notes de conférence, s.d., 1 f. ms. 


\section{Entretien}

«L'âge classique selon Robert Mandrou : une Europe des princes et de la raison d'État »: un entretien entre Robert Mandrou et Claude Mazauric, 7.5.1977, 5 f. dactyl. ; 1 lettre de Claude Mazauric, 27.5.1977.

\section{Publications}

\section{Manuscrits des ouvrages}

11 Sur l'ensemble des ouvrages publiés par Robert Mandrou (une douzaine), seuls six textes manuscrits ont été retrouvés.

« Histoire de la civilisation française » (Paris, A. Colin, 1958) : chapitre XVIII : Civilisation française, civilisation européenne, civilisation mondiale : texte manuscrit refondu et réécrit en 1976, 75 f. ; copie dactylographiée, 58 f.

"La France aux XVII ${ }^{\mathrm{e}}$ et XVIII ${ }^{\mathrm{e}}$ siècles " (Paris, Presses universitaires de France, 1967) : texte dactylographié avec corrections manuscrites, [1966], 342 p. (texte d'édition).

«Des humanistes aux hommes de science» (Paris, Ed. du Seuil, 1973) : texte manuscrit avec corrections manuscrites, $229 \mathrm{f}$.

«Louis XIV en son temps, 1661-1715» (Paris, Presses universitaires de France, 1973) : texte manuscrit avec corrections manuscrites, $547 \mathrm{f}$., plans de chaque chapitre, $12 \mathrm{f}$. ms.

« Raison et raison d'État (1649-1775) » = ) Vernunft und Staatsräson » (Berlin, Propyläen Verlag, 1976) : texte français manuscrit, $323 \mathrm{f}$., schémas de chaque chapitre, $51 \mathrm{f} . \mathrm{ms}$. ; copie dactylographiée avec corrections manuscrites, $378 \mathrm{f}$., ajouts manuscrits, $6 \mathrm{f}$., ajouts dactylographiés, $7 \mathrm{f}$; ; texte allemand dactylographié, $451 \mathrm{f}$.

«Abraham de Wicquefort: chronique discontinue de la Fronde (1648-1652), choix de textes, introduction et présentation, annotation » (Paris, Fayard, 1978) : texte manuscrit, $35 \mathrm{f}$. $36 \mathrm{f}$. (manque le $1^{\mathrm{er}}$ texte), 13 fiches ms.; copie dactylographiée, $253 \mathrm{f}$. (les 47 premières pages manquent, soit l'introduction).

«Possession et sorcellerie au XVII ${ }^{\mathrm{e}}$ siècle : textes inédits» (Paris, Fayard, 1979) : copie dactylographiée des textes, 274 f. ; exemplaire photocopié avec corrections manuscrites, $80 \mathrm{f}$.

Réédition de "Classes et luttes de classes en France au début du XVII siècle " : réédition effectuée par l'université de Nanterre en 1969 après la destruction de l'original à Florence, ronéot. p. 1 à 88 et 117 à 125.

[«Les 7 jours de Prague: 21-27 août $1968 »$ (Paris, Anthropos, 1969): plan et présentation, $7 \mathrm{f}$. ms. ; photographies de l'exemplaire original publié en Tchécoslovaquie, 48 p. impr., correspondance].

Josef Macek, « Histoire de la Bohême », (Paris, Fayard, 1984) : texte dactylographié avec corrections manuscrites de R. et Chr. Mandrou, $407 \mathrm{f}$., chronologie des princes et bibliographie, $6 \mathrm{f}$. (exemplaire de pages dactylographiées); exemplaire dactylographié avec corrections manuscrites, $270 \mathrm{f}$.

Josef Macek, «[La Renaissance à travers le miroir des siècles]»: texte dactylographié avec corrections manuscrites, $258 \mathrm{f}$.

Découpage du Pèlerin perdu, film réalisé par Guy Jorré, produit par Les éditions de l'Eolienne, Paris, conseil historique : Robert Mandrou : $66 \mathrm{f}$. dactyl. 


\section{Articles publiés} représenter l'ensemble de ses contributions aux différentes revues scientifiques (la bibliographie, présentée en annexe, recense 128 articles et 172 comptes rendus).

Textes manuscrits (39 p.) et dactylographiés de comptes rendus pour Annales ESC, 1957, Revue historique, 1970, L'Année sociologique, 1975, 1977, Historische Zeitschrift, 1978, 119 f. ; notes bibliographiques et comptes rendus, s.d., $11 \mathrm{f}$. copie dactylographiée.

"Un grand débat: les mouvements populaires et la société française du XVII ${ }^{e}$ siècle ": texte, copie dactylographiée, avec corrections manuscrites, 1959, $12 \mathrm{f}$.

"La pratique religieuse catholique en France au XviI siècle: bilan de recherches et positions de problèmes » : texte, copie dactylographiée, 1963, $22 \mathrm{f}$.

"Stimulants et freins au développement du capitalisme en France (XVI ${ }^{\mathrm{e}}-\mathrm{XVII}{ }^{\mathrm{e}}$ siècle) »: texte de la communication du colloque de Bloomington, copie dactyl., 1968, $9 \mathrm{f}$.; "Stimulants et freins au développement du capitalisme en France jusqu'à la fin du XVII siècle ", note, copie dactylographiée, $3 \mathrm{f}$.

«L'histoire: objet et méthodes »: texte, copie dactylographiée, 1970, 21 f., corrections manuscrites.

«Histoire des mentalités »: texte, copie dactylographiée, 1970, $14 \mathrm{f}$; ; tiré à part Encyclopaedia universalis, Paris, 1970, t. 8, p. 424-429, 436-438.

«Les fondations pieuses dans la ville d'Augsbourg à la fin du XvI siècle » : texte, copie dactylographiée, 1973, 38 f. (+ 1 photocopie).

"Clergé tridentin et piété populaire : thèses et hypothèses » : texte manuscrit, 1976, 12 f.; texte dactylographié, 15 f. (+ 1 copie dactylographiée).

«La poule de Carnaval: rencontres entre historiens et ethnologues »: correspondance avec Lucienne Roubin; plan, 1 f. ms. ; notes de travail, 6 f. ms. ; texte manuscrit, 1976, $14 \mathrm{f}$. ; texte, copie dactylographiée, $11 \mathrm{f}$.

«La Réforme française avant Calvin»: communication, Leipzig, 1977, texte manuscrit, $7 \mathrm{f}$. ; texte dactylographié, $5 \mathrm{f}$.

«Abraham de Wicquefort et le duc August (1646-1653): sur les relations intellectuelles entre France et Allemagne, un siècle avant les Lumières » : texte manuscrit, 1978, 36 p.; texte, copie dactylographiée, $82 \mathrm{p}$.

« France » (présentation historique) : texte, copie dactylographiée, $73 \mathrm{f}$.

«Hérétiques méconnus du xvI ${ }^{e}$ siècle européen »: texte manuscrit, [1978], 6 f.; copie dactylographiée, $6 \mathrm{f}$.

« De la modernité » : texte manuscrit, [1978], 3 f. ; copie dactylographiée, 3 f.

«L'historiographie des minorités en France : bilans et positions de problèmes » : épreuve imprimée, 1980, 18 p.

\section{Articles non publiés}

Articles rédigés, prêts pour la publication, dont les références ne nous sont pas connues.

(sans titre) : texte présenté au colloque de Monaco, 1965, texte, copie dactylographiée, $9 \mathrm{f}$. «France : histoire politique de 1500 à 1789 » : texte, pour les éditions [Garzanti], copie dactylographiée, $17 \mathrm{f}$.

« France : histoire culturelle de 1500 à 1789 » : texte, copie dactylographiée, $20 \mathrm{f}$.

Les Cahiers du Centre de Recherches Historiques, 18-19 | 1997 
«Cardinal de Richelieu (1585-1642)»: [Enciclopedia europea, Garzanti ?], texte manuscrit, $4 \mathrm{f}$. ; texte dactylographié, $4 \mathrm{f}$.

«Histoire des mentalités, dimension nécessaire de l'histoire politique»: texte pour [Schmidt], copie dactylographiée, $13 \mathrm{f}$.

« Mythologie et politique : l'exemple de Louis XIV » : texte manuscrit, s.d., $6 \mathrm{f}$.

«Culture orale et culture écrite en France aux XVII et XVIII ${ }^{e}$ siècles » : texte manuscrit, s.d., 9 f. ; texte dactylographié, $8 \mathrm{f}$.

«Louis Dermigny, historien des mentalités: la mythologie américaine, hier et aujourd'hui »: plan; texte manuscrit, s.d., $12 \mathrm{f}$; ; texte dactylographié, $13 \mathrm{f}$. (+1 copie dactylographiée); correspondance.

«Les conditionnements de la vie littéraire : aspects sociaux et institutionnels de la vie littéraire »: texte manuscrit, pour les éditions Garzanti, 1967, $90 \mathrm{f}$., $10 \mathrm{f}$. de notes bibliographiques.

«De Jeanne d'Arc à Louis XIV ", "De la Régence aux Trois Révolutions » : préfaces à la réédition de l'Histoire du peuple français, 1978, textes manuscrit, $69 \mathrm{f}$. 1 exemplaire, copie dactylographiée, 70 f. ; lettre de L. H. Parias, 31.3.1978.

"Culture savante et culture populaire en France au XVII ${ }^{e}$ siècle » : texte pour L'Histoire, 1979, 4 f. ms., exemplaire dactylographié, 4 f.; lettres de Michel Winock, 21.11.1978, 8.3.1979.

\section{Projets de publication}

«Atlas culturel et spirituel : XVI ${ }^{\mathrm{e}}-\mathrm{XVIII}{ }^{\mathrm{e}}$ » : lettre de F. de Dainville, 8.11 .1961 ; cartes, notes manuscrites ; projets de plans, $12 \mathrm{f}$. ms., $8 \mathrm{f}$. ronéot.

«Le premier Port Royal » : introduction, $3 \mathrm{f}$. ms.; plan en 13 chapitres, $1 \mathrm{f}$. ms. ; plan de chacun des chapitres, $13 \mathrm{f}$. ms. ; bibliographie pour chacun des chapitres, $13 \mathrm{f}$. ms.

\section{Édition}

Ces dossiers concernent les contrats d'édition, les relevés des droits d'auteurs, les services de presse et les lettres de remerciements pour l'envoi des livres. Robert Mandrou a noté sur la couverture de chaque dossier une liste de tous les destinataires de ses envois, sur laquelle il a coché les réponses orales ou écrites.

$<$ tab_19-19_6.pdf Image9>

\section{Correspondance générale (1954-1983)}

Le classement initial de cette partie du fonds était entièrement chronologique. Nous avons opéré un reclassement alphabétique des correspondants. L'inventaire déposé aux Archives nationales comportera le nombre de lettres, leurs dates ainsi que, lorsque nous disposons des informations nécessaires, l'institution à laquelle le correspondant est rattaché. L'astérisque (*) indique que le dossier comprend plus de dix lettres; le double astérisque (**), qu'il contient plus de 100 lettres. 


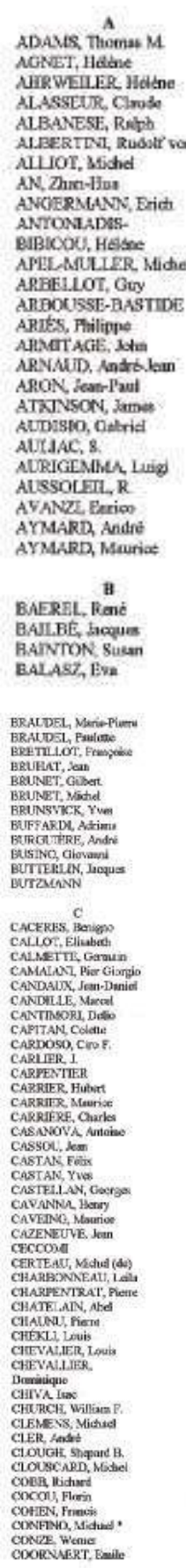

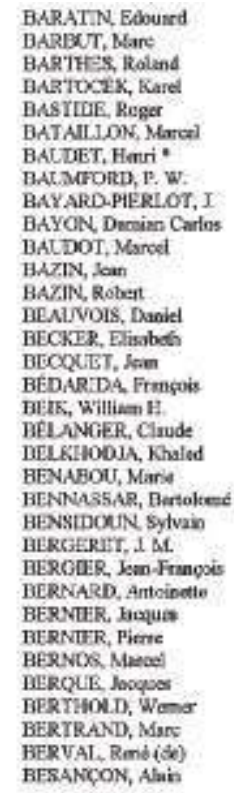

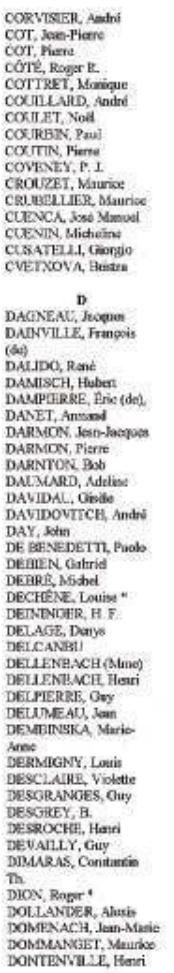

BIANCHI BANDTNELLII

Rescosio

BSBOLET, Francolis

BLACNW

BLENDINICER Frintit

a Elíinde

id, OCIL, Charies

BA, OClL, Charie

BLOCH MA

BLOCH, M. A

BOEEN TM

Boestoma

BOHORQUEZ al

BOLtLME, Generiave

BOLTANSK3, Laye

BOLLANSKS, LUS

BOCESTR, Desid I

BORST, Ams

BOUARD, Midual (de)

DOtCHakd, Cirard

BOLLOCSEAL, Mar

polvoluke Man

bolrbi, hide

BOLRDE', Clande

BOURDIEL, PArr

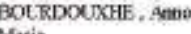

Maris:

BOLR,GFOLS, Mare

BoLrrucie, Robet

BOL VIIR, Jast

BPANDT, willy

BRANDT, Wily
REAUUDEL, Farand

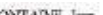

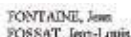

GROH, Dide

poucalin, Mastat

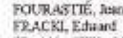

FEANCAsTiL, Pion

PRANZ, Oanti

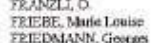

TreET, Rrangos

GACHE, 5 te

GACHE, sylwe

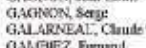

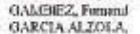

GARCN, Buligpe
GARDES, Mautio

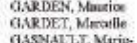

GMUE, M.

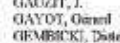

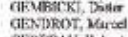

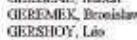

GERSTOW, Lä

GEESCHER ing

GIEYS2TOR, Nibs
GILET, Matud

aINs3irRa, Cirb

Girsura, Mimosel

GLASSCN, Sursere

GLELNSSON , Jan

CODECHCT, Һиху

colosiun Nos

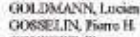

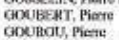

GRnusida, Anstray

GRAERS, Llom

ORALIS, Frationk
CREW, Raymond

GiRCOCHIISKA Autani

Grosser, Ailins

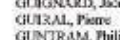

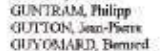

H
HALAWACHS, Piare
HAILGCRKK R. E

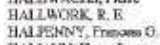

BANELAN, Jat

AAMSCHER, Abort

BARTMUNN, Framsoist

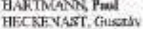

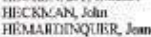

HEM⿻日,

HENTTSGEEN Gus

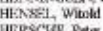

HERESCEE, PAde
HERVE, Fom

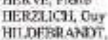

Finderd

HIRSCKL BGimow
HIRSCBRA

Honsenumac Eac

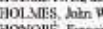

HONORE, een

HOERT Todito

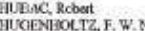

HUCGENHOLTZ, E. W

sanitum, yoos

L.10u, Mitippe

DABEX": Nariox

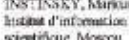

s.

AaESBER, Han

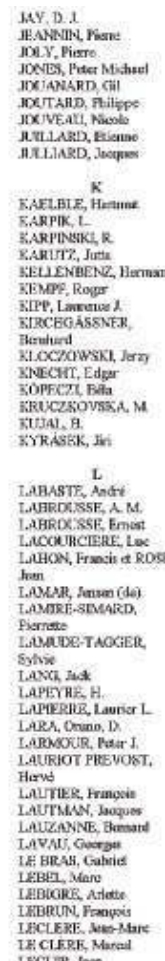

MY. 1 L 1 ,

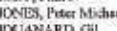

Dertand, Giltp

R.LiNRD, Juecps:

AELAIE, Heat

ENAEN2, Herm

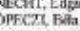

As 8 , ik

ADTE, Antit
RDESSE, A

OOURCEERE, Lo

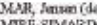

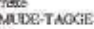

sore, aus

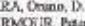

AITIER Frimosin
AUTMMN, boques

V.AN, Goors

BEA, Mano

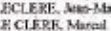

IFCLE, Jeen 


\begin{tabular}{|c|c|c|c|}
\hline 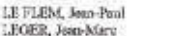 & MAURO, Hoblete & $\begin{array}{l}\text { Puovi, lean } \\
\text { Panst }\end{array}$ & CIVISIER 3 \\
\hline 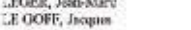 & YAEDUBCCITZ, Heliont & 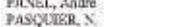 & QUERE, At: Rey,mod \\
\hline LEINAER, Eadry & MELCHEN-BONVEET, & PIARCE, Bhin & GLEREART, Jat \\
\hline TMEXI. FaA & Satkine & FELLETIER, JeandGey & QULIET, Emord \\
\hline L.F MOCEL, Marie & MEJCHOR-3ONNET, & FELIETIER, Whe & \\
\hline 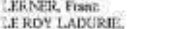 & On & PERINAr, Adoss & 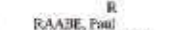 \\
\hline $\begin{array}{l}\text { F RDY LADCIAIE, } \\
\text { Ensumbel }\end{array}$ & 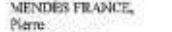 & 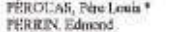 & 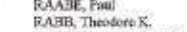 \\
\hline LFECO,tT, Jalian & AIRIEY, hen & PERROT. Sfideles & 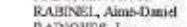 \\
\hline IFSNODORSKL, & MESLEN, Machel & FikikouK, Frmpess & RNDioyss, I \\
\hline 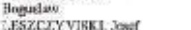 & MESSEVER, Didir & FERROYY, EAnanI & 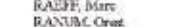 \\
\hline 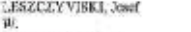 & 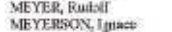 & 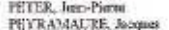 & 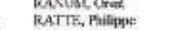 \\
\hline EuLAOT, Рial * & MEYLA', Heid & 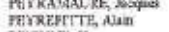 & ВАYЗUT, Pwal \\
\hline WNES Reme & MaGGNOr, Gtant & PEYrinT, it & 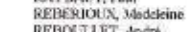 \\
\hline LEYY-LAOTES, & MINNDER, Rojat & PYELPEE & REBOCLLET, indin \\
\hline 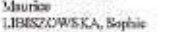 & 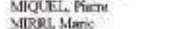 & 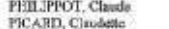 & 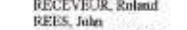 \\
\hline 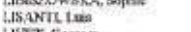 & MIITLEH, be & 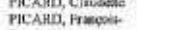 & BEGEMQETER, JeN- \\
\hline & & 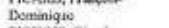 & Louis (rma) \\
\hline 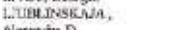 & АOL HO, Mañ ice & 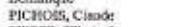 & 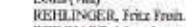 \\
\hline Aliectrids D D & MOLNAR, In & PIEPER, Elisibsth & REINAND, Maroed \\
\hline LOSARRD, Naurix & MONTEYORTE, Tolow & PIIERRARD, $\mathrm{P}$. & BEISs, Fanoties \\
\hline 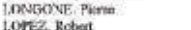 & MDNTER, WHAllam & PIETRZAK- & 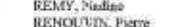 \\
\hline 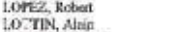 & 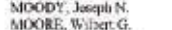 & PCWLOWSEK, loos & 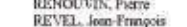 \\
\hline WOZK, Gerhant & 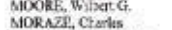 & $\begin{array}{l}\text { PETTERSAMSOR, } \\
\text { Chritine }\end{array}$ & 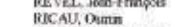 \\
\hline WELONSEY, Madini & 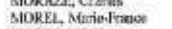 & $\begin{array}{l}\text { liriatine } \\
\text { ploNor, Heri }\end{array}$ & RMDENUALER, ENW \\
\hline WIT AUD, Oiviar & MORDS Alfist & & RaD, Jocestie \\
\hline 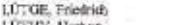 & MORDN, Manitios & PlivKotY, Devis H & RENIERK Gorpes-Herai \\
\hline LITFY, Herpas: & MORNAfAU, Mithel & PIORECW'SKL, kns & \\
\hline UIJZ, Heinrid & MOLLRNAS, Karé & PTIZ, Emol & BOBNBON, Filip E I \\
\hline 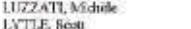 & MROCZKOWSE, & PIUK, Ambeskanir & $\begin{array}{l}\text { ROCHE, Danie } \\
\text { DOGE, }\end{array}$ \\
\hline LVTLE, SEOA & 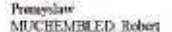 & 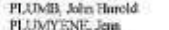 & 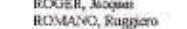 \\
\hline M & & Poessov, Goronger & BOMOH, Chizitita \\
\hline MACEK, Jeed ". & $\begin{array}{r}\mathrm{V} \\
\mathrm{s}\end{array}$ & Howex Lio & HONCAYO1O, Maroo \\
\hline MACHE.FER, Th. & NADON, Fime & POL AKON, Len & BOOVER, Rrsnomd (di) \\
\hline MNe AN, Outawe & 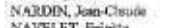 & POUNIIZ, Q Fotithe & 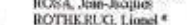 \\
\hline MAL AURTE, Aan & NAVFLST, Erigite & (ucen) & \\
\hline 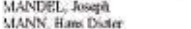 & 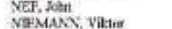 & 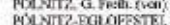 & HOWLAND, Rolen \\
\hline MARCZEW'SKA, Jar & 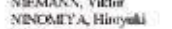 & 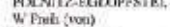 & HOYDE-SART, $x$ \\
\hline MASElN, Mirelle & NeSH, Cameron & POMGatit, Rest & \\
\hline $\begin{array}{l}\text { MARRRUS, MSdhad II. } \\
\text { MARTS }\end{array}$ & SFORA, Tiane & 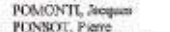 & 5 \\
\hline 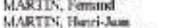 & 0 & $\begin{array}{l}\text { PONSO, Pere } \\
\text { POPIST:LNU, Crisian }\end{array}$ & SARTTA, Armando * \\
\hline MARTSEAL, Frapois & OKaMOTO, Acha & PCRCINIEV, Bonix & SACACOS, Fien \\
\hline 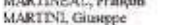 & OrCiBdi, Jan & POULX, Jes:Cloude & SANASOC, Lowat \\
\hline MASCHEI, Brist * & OREOCTOONL, Hiars & PREPOSIET, han & 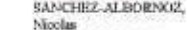 \\
\hline Masst. Prime & 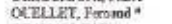 & FEEYDT, D & 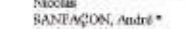 \\
\hline MASSTCOTIE, Guy & OEANAMS Didiet & PREVBario, Marí or & SANFACON, Bastased \\
\hline $\begin{array}{l}\text { MASTELLONE, Salvo } \\
\text { MATHES Par }\end{array}$ & $\mathbf{P}$ & 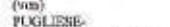 & BQNTE \\
\hline MATHEU, joopes P. & PACH 2sigmond Pal & CRRRCTELLL Givaneni & 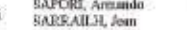 \\
\hline MNUDSINDER, , NLOE L L & BNesi, hemstio & pUasaur, Halinat & 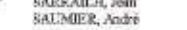 \\
\hline & \multirow{2}{*}{\multicolumn{2}{|c|}{ Wrsepents, H }} & $y$ \\
\hline WA.UAT, Jan-Piane & \multirow{2}{*}{\multicolumn{2}{|c|}{$\begin{array}{l}\text { WITIMAN, Tibor } \\
\text { won } F \text {, Antin a furs }\end{array}$}} & YAHCDEN, Mtyram. \\
\hline WATELET, Hulber. & & & \\
\hline WEAEK, Eugen & \multicolumn{2}{|c|}{ WoefF, Alsilipse } & $x$ \\
\hline WEgles, Eenuasin & \multicolumn{2}{|c|}{ WDLF, surs } & ZABABEXCI, Pmols \\
\hline WETUER, Jou & \multicolumn{2}{|c|}{ WYMOCH, Isse } & ZANGHIERL Reode \\
\hline WEINEAR, Dern Mieres & \multicolumn{2}{|c|}{ WOLOSZYNSE, } & ZAYXIEW, Adet \\
\hline Wres, theturd & \multirow{2}{*}{\multicolumn{2}{|c|}{ Bysard }} & ZEREOCNTAV, Tinsen \\
\hline Wru UIRs, Gexrges & & & 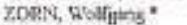 \\
\hline WELLMGANN, IREE & \multicolumn{2}{|c|}{ WYCZANESK, Antrog } & ZYSBEEGG, Aadre \\
\hline WrRNARR, Vidise & \multicolumn{2}{|c|}{ WYTII, Lamatoo } & \\
\hline
\end{tabular}

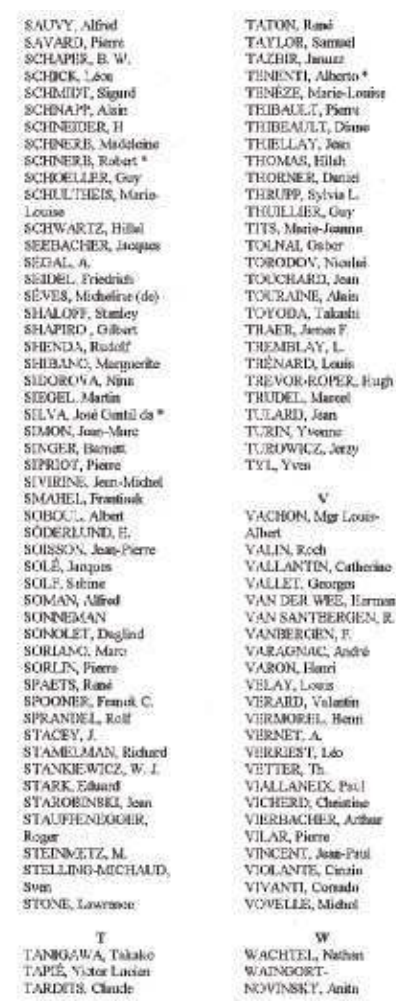

Témoignages, notices nécrologiques, correspondance (1984-1997)

Réception en hommage à R. Mandrou par l'ambassadeur du Canada, 1986: invitation, télégramme, copie du discours de Pierre Goubert lors de la réception, $2 \mathrm{f}$. dactyl., correspondance ; copie d'une ébauche de la préface aux Mélanges de Philippe Joutard et Jean Lecuir, $13 \mathrm{f}$. dactyl.

Allocutions et témoignages : textes dactylographiés rassemblés à l'issue de la " journée Robert mandrou " organisée à l'occasion du don de ses archives (juin 1995) : Jacques Chevallier, Christiane Mandrou, Lucienne Roubin, Arlette Farge, Philippe Joutard, Jean Lecuir, « Robert Mandrou à Nanterre-Paris X »; Etienne François, « Robert Mandrou : une figure exemplaire »; Henriette Asséo, « Hommage à Robert Mandrou ».

\section{Lettres de Robert Mandrou remises par ses correspondants}

Cette partie du fonds est en cours de constitution.

17 Myriam Yardeni, professeur, département d'histoire générale, université de Haïfa, 16 lettres et cartes, janvier 1963-juillet 1968. Jean Lecuir, 6 lettres et carte, octobre 1967- 
juin 1982. Etienne François (photocopies), 14 lettres, septembre 1974-août 1980. Les lettres de Robert Mandrou à Lucien Febvre, qui lui ont été restituées par Suzanne Febvre, sont restées dans le dossier Annales. 50 lettres de Robert Mandrou à ses parents lors de son séjour dans l'établissement médical de la rue Boileau à Paris, 11 juillet 1956-26 août 1957. 